\title{
Pre-print
}

Tan, B. and Otay, E., "Modeling and Analysis of Vessel Casualties Resulting from Tanker Traffic through Narrow Waterways,” Naval Research Logistics, Vol. 46, No. 8, pp. 871-892, 1999.

\section{MODELING AND ANALYSIS OF VESSEL CASUALTIES RESULTING FROM TANKER TRAFFIC THROUGH NARROW WATERWAYS}

\author{
Barış Tan \\ Graduate School of Business, Koç University \\ Çayır Cad. 80860 Istinye Istanbul, Turkey \\ btan@ku.edu.tr \\ Emre N. Otay \\ Civil Engineering Department, Boğaziçi University \\ 80815 Bebek, Istanbul, Turkey \\ otay@,boun.edu.tr
}

May 1999

\begin{abstract}
In this paper, we present a physics-based stochastic model to investigate vessel casualties resulting from tanker traffic through a narrow waterway. A state-space model is developed to represent the waterway and the location of vessels at a given time. We first determine the distribution of surface current at a given location of the waterway depending on channel geometry, bottom topography, boundary conditions, and the distribution of wind. Then we determine the distribution of the angular drift for a given vessel travelling at a given location of a waterway. Finally, we incorporate the drift probabilities and random arrival of vessels into a Markov chain model. By analyzing the time-dependent and the steady-state probabilities of the Markov chain, we obtain risk measures such as the probability of casualty at a given location and also the expected number of casualties for a given number of vessels arriving per unit time. Analysis of the Markovian model also yields an analytical result that shows that the expected number of casualties is proportional to square of the tanker arrival rate. We present our methodology on an experimental model of a hypothetical narrow waterway.
\end{abstract}

Keywords: Stochastic models, Markov chain models, analytical and numerical modeling techniques, casualty models, transportation, risk analysis 


\section{Modeling and Analysis of Vessel Casualties Resulting From Tanker Traffic Through Narrow Waterways}

\section{Introduction}

Vessel casualties resulting from oil tanker traffic may have important economic and environmental consequences. Especially, if a casualty yields an oil spillage, environmental effects threaten the local ecology while its economic effects may ruin the ship-owner, and even, the liability insurer (Unsworth, 1997; Unsworth, 1996; Oshins, 1992; Shapiro and Huntley, 1989). Furthermore, if the casualty takes place in a waterway, the traffic through a waterway may be blocked for a long period of time affecting the other vessels. Since 1960, there have been 1720 oil spills resulting in a spill volume of around 1.8 billion gallons of oil in the world (Etkin, 1997).

Our study is motivated by the foreseen increase in the oil tanker traffic through the Bosphorus Strait as a result of exploitation of oil reserves in the former-Russian republics. This paper is a first step towards the modeling and analysis of vessel casualties resulting from tanker traffic through the Bosphorus.

In this paper, we present a physics-based stochastic model that can be analyzed to estimate the vessel casualties from tanker traffic through a narrow waterway, such as the Bosphorus, the Dardanelles, the Houston Ship Channel, the Mississippi River, or the Suez Canal.

Vessels travelling through a narrow waterway usually follow a designated course. However, the actual position of a vessel may differ from its intended route at a given location and time. This is a result of the hydrodynamic conditions, i.e., currents and waves, visibility, wind, geometric constraints, and the boat traffic at that location and time. If the actual position of a vessel differs from its intended route, i.e., if the vessel drifts, it may cause an intervessel collision with another vessel drifted to the same location at the same time. We incorporate the variability in physical forcing mechanisms and the randomness of the vessel arrivals in our stochastic model.

We develop a state-space representation of a waterway and the location of vessels at a given time. We then revise the intended route of a vessel to a probability distribution that incorporates the effects of possible drift depending on the physical properties of the vessel such as its mass, length, and draft. We incorporate these probabilities and random arrival of vessels into a Markov chain model. By analyzing the time-dependent probabilities of the Markov process, we obtain performance measures such as the probability of casualty and the expected number of casualties.

The model provides the risk charts that show the casualty probabilities across the geometry of the waterway at a given time and vessel traffic intensity. Furthermore the model can be used to investigate the relationship between the vessel traffic intensity and global measures of casualty risk such as the expected number of casualties per vessel or per time.

The contribution of this paper is threefold. First a new physics-based stochastic model is introduced to study the tanker accidents in narrow channels. Second, an analytic formula that relates the accident risk to the vessel traffic is derived. Third, an experimental model is developed and applied to a hypothetical waterway. 
The organization of the remaining part of this study is as follows. In Section 2, we review the pertinent literature on modeling casualty risk. In Section 3, the general model is introduced. Hydrodynamic model is discussed in Section 4 and modeling of drift is explained in Section 5. The Markov model of casualties is presented in Section 6. Steady-state analysis of vessel casualties is given in Section 7. Analysis of a special case and related numerical results are presented in Section 8. Finally conclusions are given in Section 9.

\section{Past Work}

Most of the studies on modeling casualties are based on statistical estimation methods and time-series analysis that utilize past data. Some of these studies are found in econometric safety analysis literature.

Talley (1995a) analyzes the determinants of accident severity to evaluate the policies for reducing the vessel damage severity and the subsequent oil spillage of tanker accidents. Talley (1995b) investigates the determinants of accident passenger-vessel damage cost. Anderson and Talley (1995) use a similar approach to study the determinants of the oil cargo spill, size of tanker and tanker barge vessel accidents. Talley (1996) investigates the determinants of the risk and the severity of cargo of containership accidents by using microdata of vessel accidents in an eight-year period. Cohen (1995) presents a market model to evaluate the economic losses of the 1989 Exxon Valdez oil spill. Le Blanc et. al. (1988) present a regression model to estimate the vessel casualties depending on river stage, system usage rate, and weather. Le Blanc et. al. (1995) present a cluster analysis of vessel accidents on the lower Mississippi River.

The closest studies to the problem are the ones developed by the United States Department of Transportation for the U.S. Coast Guard's Office of Navigation Safety and Waterway. Maio et. al. (1991) develop a regression model to estimate the waterway casualty rate depending on the type of waterway, average current velocity, visibility, wind velocity, length of primary traffic route, channel width, etc. Kornhauser and Clark (1995) use this regression model to estimate the vessel casualties resulting from additional oil tanker traffic through the Bosphorus.

We believe that methods based on statistical estimation are not adequate to assess the casualty risks due to two main reasons. First, the data collected over a time period at a given waterway cannot be used immediately to analyze the risks at another waterway since the physical forcing mechanisms, vessel traffic, etc. may be very different. Second, if the vessel traffic is expected to increase rapidly in the near future, it is not possible to build a reliable estimation model based on the previous data since such a condition has not been observed in the past.

In the present study, our objective is to develop a mathematical model of vessel casualties and then use this model to estimate the casualties. As far as we know, there is no physics-based stochastic model developed to investigate vessel casualties. Our model uses the effects of forcing mechanisms that determine the intensity and direction of currents, waves, etc., wind, visibility, traffic intensity and other factors and provides timedependent estimates of vessel casualties at a given location of the waterway, as well as, global risk measures. Therefore our model can be used both in planning and policy development, and also day-to-day operation of the traffic through the waterways. 


\section{General Model}

The ex-president of the Turkish Marine Pilots Association of the Bosphorus, Capt. Oğuzülgen identifies the main causes of accidents in narrow straits as the natural geometry of the strait, surface currents, restricted visibility, and local traffic (Oğuzülgen, 1995). In our physics-based model, we capture all of these factors. Our model consists of three building blocks. Figure 1 below depicts these building blocks and their interaction.

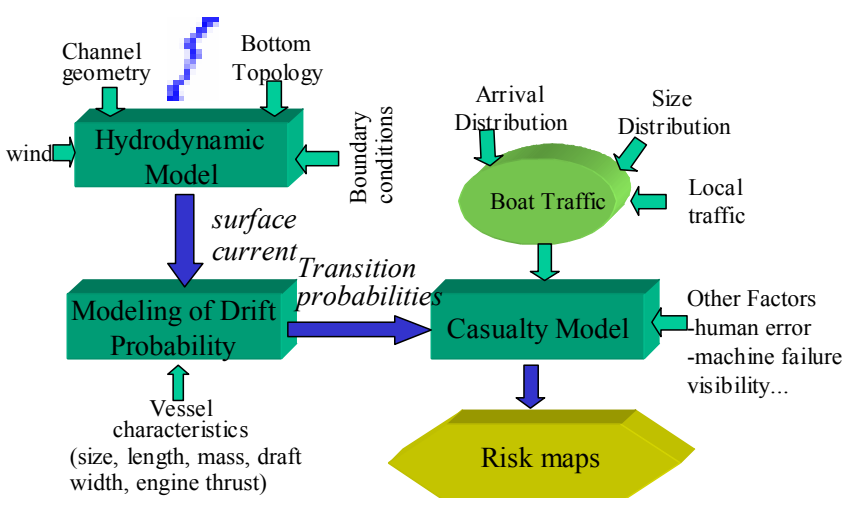

Figure 1. General Model

The first building block is the hydrodynamic model. The most important forcing mechanism that affects vessels travelling on a waterway is the surface current. This model determines the current velocity at a given location of the waterway depending on wind, channel geometry, bottom topography, and boundary conditions. In order to ensure numerical tractability, a waterway is represented as a grid consisting of a finite number of elements. The first building block yields the current velocity in each of these elements.

The second building block gives the drift probabilities for a given vessel travelling at a given location in a waterway. Because of surface current, a vessel may drift from its original route. This drift is one of the most important factors for vessel casualties along a waterway. The effect of surface current on a vessel will be different depending on the characteristics of the vessel, i.e., depending on its size, length, draft, mass, engine thrust, etc. This building block first determines the distribution of drift from a vessel's original route and then discretizes this distribution to three drift probabilities. For example, for a vessel located in a given grid and travelling north, this model estimates the probabilities that there will be no drift, or a drift to the northwest or northeast grids during the next time period depending on the characteristics of the vessel and also the current velocity in this grid.

The third building block is a state-space model of vessels travelling along a waterway. This model incorporates the drift probabilities obtained from the second building block, arrival distribution of the vessels, and other effects into a Markov chain model. In this block, we do not differentiate vessels according to their type, length, etc., and carry our analysis for a typical vessel. However, the properties of ships, such as their length, mass, draft, etc. are taken into consideration in the previous building block where the drift is modeled. These characteristics are reflected in the drift probabilities we use in this block. Alternatively, one can model each vessel according to its properties and perform the state space analysis accordingly. In this case, drift probabilities of the state space model will depend not only on current direction and velocity at a given grid but also the vessel type. This will increase the number of parameters to be estimated dramatically. Therefore, this 
part of the model is carried out for a typical vessel and the differences among vessels are reflected in drift probabilities.

The probabilities that an intervessel collision or a grounding takes place at a given location at a given time are derived by analyzing the probability distribution of vessel positions in the waterway at a given time. Finally, steady-state probability distributions and expected number of intervessel collisions, groundings, and total casualties are obtained. These results allow us to construct various risk charts. A major part of this paper is on the casualty model where operations research techniques have been used. In the next sections, we give more detailed information about these building blocks.

\section{Hydrodynamic Model}

The hydrodynamic model determines the surface current velocity at a given location of the waterway depending on wind, channel geometry, bottom topography, and boundary conditions as shown in Figure 2 . We assume that the effect of currents on the vessel drift is more profound compared to the effects of waves and other physical forces in a narrow waterway.

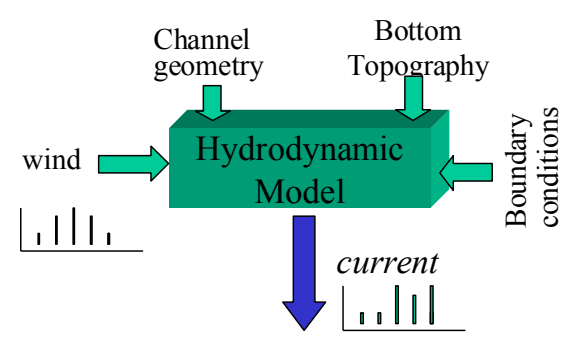

Figure 2. Hydrodynamic Model

In the hydrodynamic model, the current circulation is governed by the continuity (conservation of mass) and Navier-Stokes equations (conservation of linear momentum). Using the appropriate boundary conditions, the governing equations are numerically solved to determine the surface current velocity at a given location of a waterway. A finite element model is developed for numerical solution of the hydrodynamic system where the waterway is represented as a curvilinear grid. Equations (1)-(3) list the equations that are used in the finite elements model for shallow water current circulation.

$$
\begin{gathered}
\frac{\partial \eta}{\partial t}+\frac{\partial}{\partial x}(u h)+\frac{\partial}{\partial y}(v h)=0 \\
\frac{\partial u}{\partial t}+u \frac{\partial u}{\partial x}+v \frac{\partial u}{\partial y}=-g \frac{\partial}{\partial x}(h+\eta)-\frac{\epsilon_{x x}}{\rho} \frac{\partial^{2} u}{\partial x^{2}}-\frac{\epsilon_{x y}}{\rho} \frac{\partial^{2} u}{\partial y^{2}}+\frac{1}{\rho h}\left(\tau_{s x}-\tau_{b x}\right), \\
\frac{\partial v}{\partial t}+u \frac{\partial v}{\partial x}+v \frac{\partial v}{\partial y}=-g \frac{\partial}{\partial y}(h+\eta)-\frac{\epsilon_{y x}}{\rho} \frac{\partial^{2} v}{\partial x^{2}}-\frac{\epsilon_{y y}}{\rho} \frac{\partial^{2} v}{\partial y^{2}}+\frac{1}{\rho h}\left(\tau_{s y}-\tau_{b y}\right)
\end{gathered}
$$

where $t$ is time, $x, y$ are horizontal distances, $g$ is gravitational acceleration, $\rho$ is water density, $h$ is water depth, $\eta$ is water surface elevation, $u, v$ are mean current velocities in $x$ and $y$ directions, $\tau_{s x}, \tau_{s y}$ are surface wind stresses, $\tau_{b x}, \tau_{b y}$ are bottom shear stresses, and $\varepsilon_{x x}, \varepsilon_{x y}, \varepsilon_{y x}, \varepsilon_{y y}$ are turbulent eddy viscosities. The first equation above 
is the continuity equation and the next two equations are the momentum equations in $\mathrm{x}$ and $\mathrm{y}$ directions respectively. For a detailed discussion of Navier-Stokes equations, its assumptions, and parameters, the reader is referred to a fluid dynamics reference book, e.g. (Daily and Harleman, 1966).

Although channel geometry and bottom topography do not change with time, wind velocity and boundary conditions alter with time. In order to incorporate the effect of wind velocity variability into our model, we first solve the finite element model for each realization of wind and boundary conditions deterministically and obtain the corresponding current circulation. By drawing wind and boundary condition realizations from their yearly distribution, we determine the approximate distribution of current circulation. Note that incorporating the variability of wind and boundary conditions directly into the equations to determine the current velocity and then solving the resulting stochastic differential equations directly would yield the exact current distribution. However the latter method is numerically and theoretically intractable.

\section{Modeling of Drift}

After determining the distribution of current velocity, we analyze the effects of the current on the intended route of a vessel by incorporating the characteristics of the vessel into a drift model. We first derive the equations that yield the drift from a vessel's original route depending of current velocity and characteristics of the vessel including its mass, length, draft, and engine thrust. Figure 3 shows the characteristics of a vessel, current, and drift.

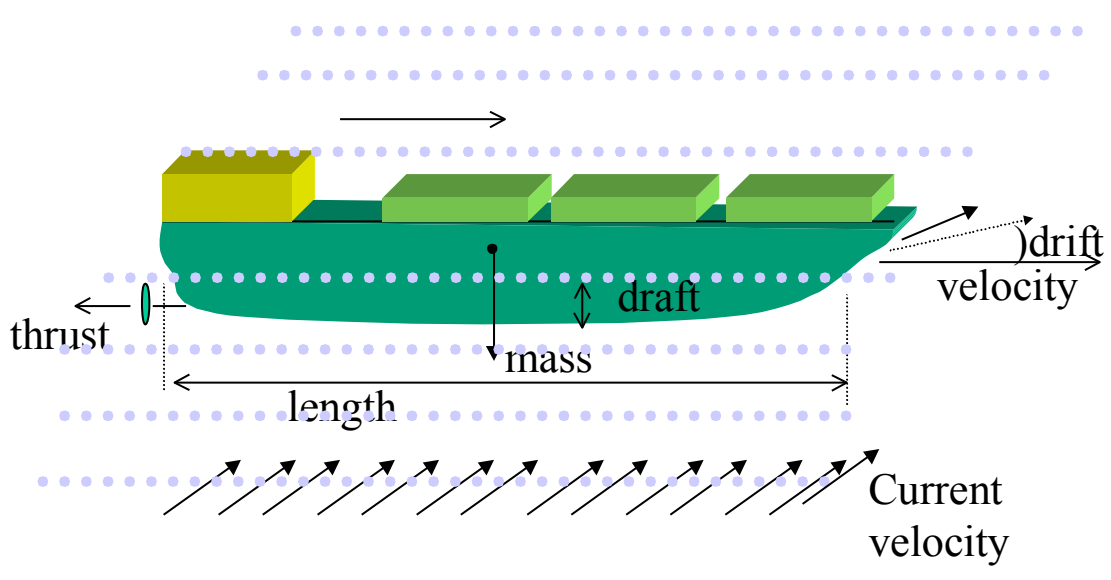

Figure 3. Modeling of Drift

Figure 4 shows the original route and the modified route of a ship as a result of the current on $x y$ plane. Conservation of energy equations are utilized to determine the angular drift from a vessel's original route. These equations describe the motion of a ship under the effect of surface current. Since our objective is to determine the state transition probabilities, the equations are solved for angular drift and angular drift velocity. 


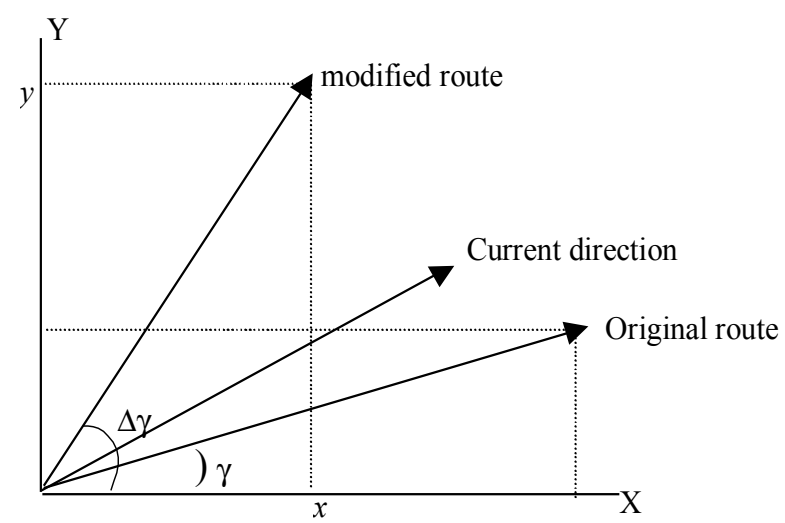

Figure 4. Original route and modified route of a ship as a result of current

Let $\Delta \gamma$ be the drift angle. From Figure 4, we can write

$$
\Delta \gamma=\tan ^{-1}(y / x)
$$

where $y$ and $x$ are the are the $y$ - and $x$-coordinates of the resulting location of a vessel under the effect of a current. Therefore the angular velocity $\omega$ is

$$
\omega=\frac{\mathrm{d}(\Delta \gamma)}{\mathrm{d} t}
$$

The sums of hydrodynamic forces acting on the ship in $x$ - and $y$-directions, $F_{x}$ and $F_{y}$, describe the motion of a ship with mass $m$ given as

$$
\begin{aligned}
& m \frac{\mathrm{d}^{2} x}{\mathrm{~d} t^{2}}=F_{x} \\
& m \frac{\mathrm{d}^{2} y}{\mathrm{~d} t^{2}}=F_{y}
\end{aligned}
$$

The sums hydrodynamic forces acting on the ship depend on the relative current and also on the cross sectional are of the ship:

$$
\begin{aligned}
& F_{x}=\frac{1}{2} C \rho A_{x}|\vec{v}| v_{x} \\
& F_{y}=\frac{1}{2} C \rho A_{y}|\vec{v}| v_{y}
\end{aligned}
$$

where $C$ is the hydrodynamic friction constant, $\rho$ is the water density, $A_{x}$ and $A_{y}$ are projections of cross-sectional areas of the ship in contact with the current in $x$ - and $y$-directions. The length, width, and draft of a vessel are used to determine the cross sectional area of the ship in a given direction. In the above equation $\vec{v}$ is the relative current with respect to ship motion that can be expressed as

$$
\vec{v}=v_{x} \vec{i}+v_{y} \vec{j}=\left(v_{x, c}-v_{x, s}\right) \vec{i}+\left(v_{y, c}-v_{y, s}\right) \vec{j}
$$

where $v_{x, c}$ and $v_{y, c}$ are current velocity components in $x$ - and $y$-directions, and, $v_{x, s}$ and $v_{y, s}$ are ship velocity components in $x$ - and $y$-directions. For more information on the derivation of motion equations, the parameters used, and assumptions, the reader is referred to a hydrodynamics reference book, e.g., Lamb (1945). 
Successive substitution and simplification of the above equations yield a set of Riccatti-type nonlinear differential equations. In these equations, current velocity, length, mass, and draft are variable while engine thrust and therefore ship velocity is constant since vessels are required to travel with constant speed along a waterway. The length, mass, and draft of each vessel travelling along a waterway are different. Therefore, the drift will be different for each vessel type and for each realization of current velocity at a given location.

In order to incorporate the effects of variability in our model, we first classify each vessel into a different number of discrete vessel type based on its characteristics. In a similar way, current velocity is also classified into a different number of realizations. Then we solve the above equations deterministically for each realization of current velocity and vessel type and obtain approximate angular drift distribution by compiling the solutions of each realization according to the frequency distribution of the vessels and current velocity realizations. After obtaining the angular drift distribution, we discretize the distribution into three drift probabilities that are used as state-transition probabilities in the Markov chain model. Figure 5 summarizes the inputs and outputs of this building block.

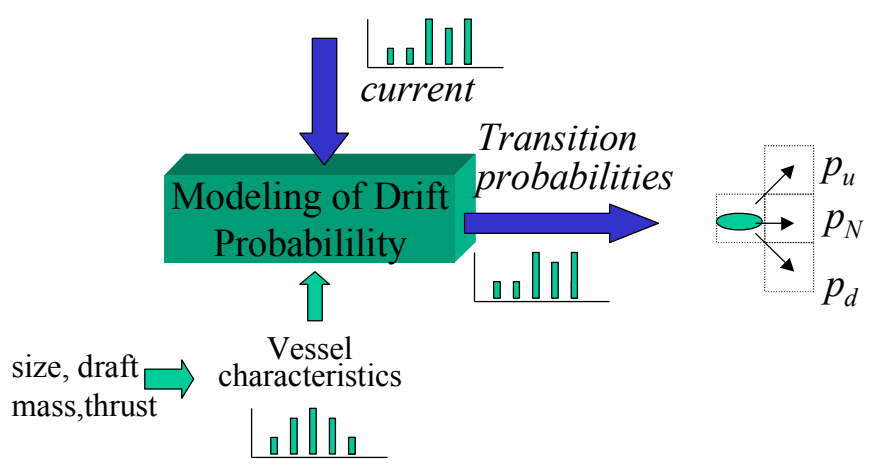

Figure 5. Modeling of Drift Probability

\section{Modeling of Casualties}

The last building block is a casualty model that incorporates drift probabilities, arrival distribution, and other effects into a Markov chain model. First, a state-space representation of the waterway is obtained. This is done by dividing each element of the grid used in the hydrodynamic model into smaller subelements to increase the accuracy of vessel movements along the waterway. Second, we model the vessel traffic by analyzing the travel of each vessel along the waterway as a random walk defined by the drift probabilities obtained. More specifically, we obtain the probability that a vessel is located at a given location of the waterway at a given time from the transient analysis of the Markov chain.

The two most important vessel casualties are grounding and collision. It is reported that $24.8 \%$ of oil spills from vessels (1960-1995) is due to "groundings" while $21 \%$ of oil spills from vessels (1960-1995) is due to "collisions" (Etkin, 1997). Therefore, only intervessel collision and grounding are modeled as possible casualties resulting from tanker traffic. The steady-state intervessel collision and grounding probabilities and also the expected number of intervessel collisions, groundings, and casualties in the long run are obtained. Risk maps and other risk charts are derived from the steady-state results. Figure 6 shows the components of the casualty model. Tan and Otay (1998) present an earlier version of the casualty model. 


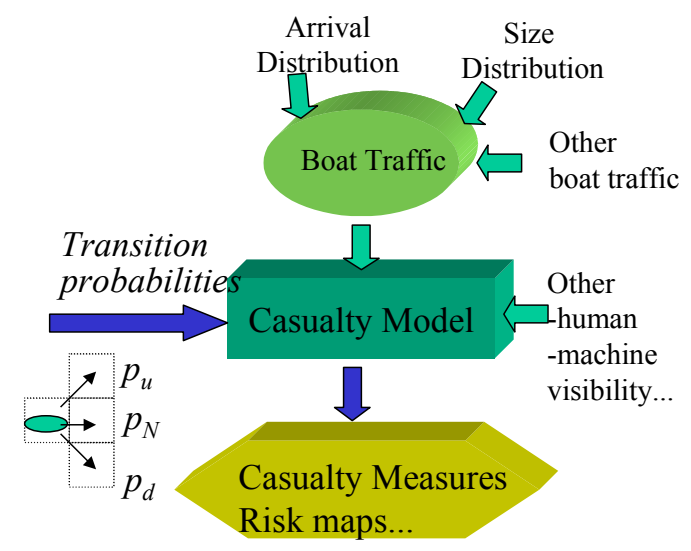

Figure 6. Modeling of Casualties

\subsection{Modeling of Vessel Traffic}

In this section, we develop a model that can be used to estimate the locations of the vessels, and therefore, to determine the casualty probabilities conditioned on the current locations of vessels. This analysis can be used in traffic control in conjunction with a system that tracks the vessel in time and locates them at a given time.

We represent the waterway as an $N \times M$ rectangular grid. Each grid represents the location of a vessel in a waterway therefore corresponds to a specific state in our state-space model. That is, our state space is $S=\{(i, j)$ : $i=1,2, . ., N ; j=1,2, . ., M\}$. Note that this representation does not assume that the waterway under consideration is rectangular. This representation transforms geographical grids to a rectangular mesh and then to corresponding states of the state space. Figure 7 shows this transformation for the Bosphorus.

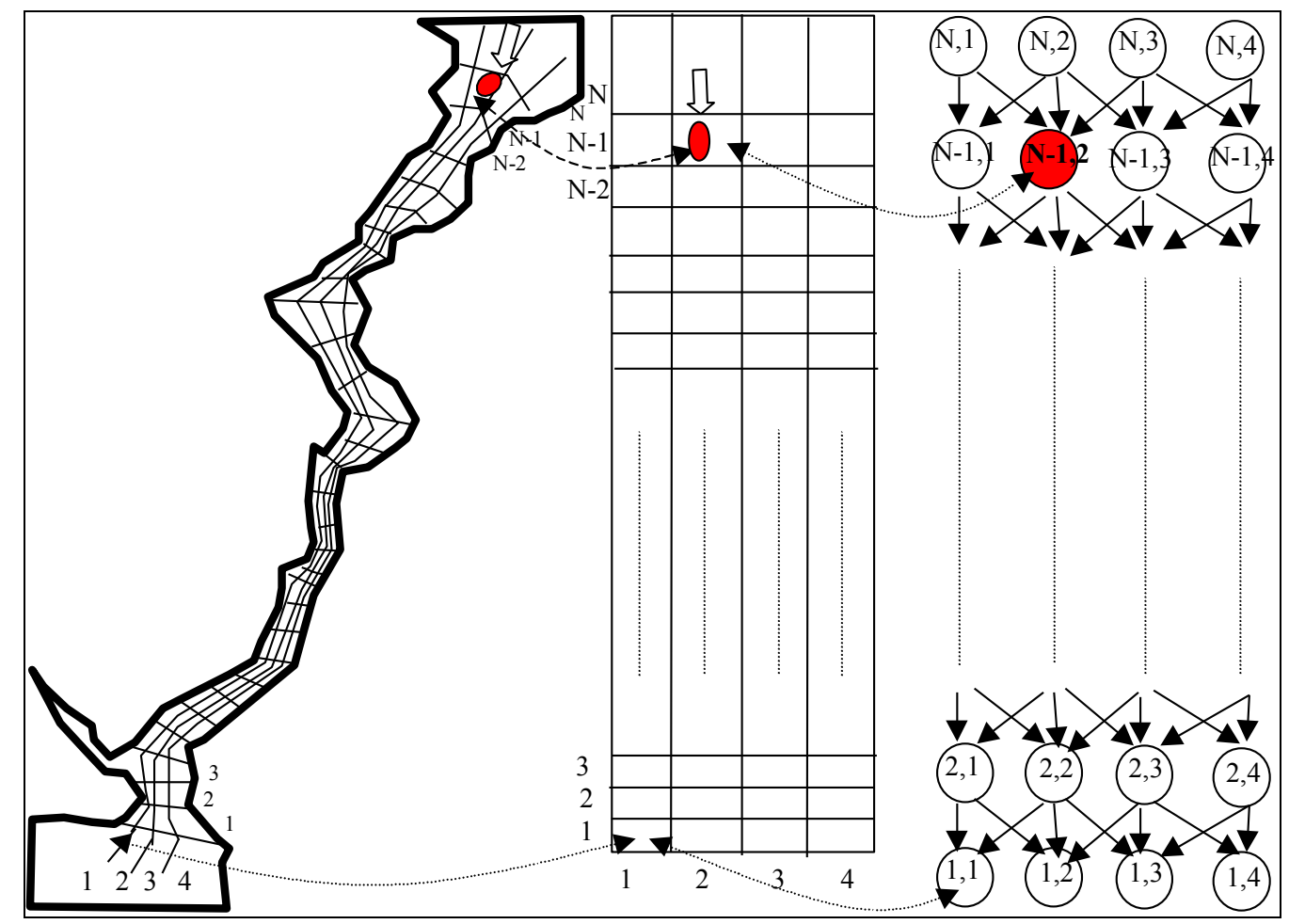

Figure 7. Representing the Bosphorus as a rectangular grid and corresponding state transition diagram for a vessel travelling downward 
Let the state of the system be observed with $\Delta t$ increments. That is, discrete time representation is assumed. All tankers travelling through a waterway are required to maintain a constant speed, say $v_{s}$ that is 10 nmiles/hr for the Bosphorus. Let the length of the waterway be $L$ units. Then the length of each grid is $L / N$ units. Since each grid represents the location of a vessel, the length of a grid must be comparable to the length of a vessel. For example, if the minimum vessel length is, say, 100 meters then the length of each grid must be greater than 100 meters, say, 150 meters. A vessel travelling with a speed of $v_{s}$ passes a given grid in $L /\left(N v_{s}\right)$ time units. This time is taken to be the sampling time $\Delta t$, i.e., $\Delta t=L /\left(N v_{s}\right)$.

Let $X_{k}^{n}(t)$ be the location of a vessel moving in direction $k, k$ is $\mathrm{U}$ for a vessel travelling up and $\mathrm{D}$ for a vessel travelling down, at time $t$ and $n$ is the time that the vessel entered the waterway. To simplify further derivations, it is assumed that at most one vessel enters the waterway during $\Delta t$ from a given direction. Therefore $X_{k}^{n}(t)$ completely specifies the state of a given vessel. If $\Delta t$ is sufficiently small compared to the interarrival times of vessels, this assumption can easily be justified.

For example, if the length of each grid is $L / N=150$ meters and $v_{s}=10 \mathrm{nmiles} / \mathrm{hr}=18530 \mathrm{~meters} / \mathrm{hr}$. This implies that $\Delta t$ is about 30 seconds; that is, the location of the vessels is observed every 30 seconds. Therefore it is also assumed that at most one vessel enters from a given direction in a period of 30 seconds. In 1997, 50000 vessels passed through the Bosphorus. Among all the vessels passed through the Bosphorus in 1997, 4303 of them were oil tankers. Then assuming that vessels can travel anytime during the year and half of them passed the Bosphorus travelling North and half of them travelling South, the average interarrival time for vessels is around 1260 seconds and that for oil tankers is 14668 seconds. For both cases, assuming at most one vessel enters from a given direction in a given 30 seconds can be justified.

Although a vessel has a specific route to follow, the actual route may be different as a result of the forcing mechanisms that depend on geometry, hydromechanics, etc. Therefore the location of a vessel at time $t$ is a random variable due to the possible deviations from the route. Therefore $\left\{X_{k}^{n}(t), t=0,1,2 \ldots\right\}$ is a discrete parameter, discrete state-space stochastic process defined on the state space $S$. Furthermore, the location of the vessel at time $t+1$ only depends on its location at time $t$. Then $\left\{X_{k}^{n}(t), t=0,1,2 \ldots\right\}$ is a Markov chain.

We assume that the movements of vessel along the waterway are independent of each other. Note that this is a simplifying assumption since vessels may alter their routings temporarily to avoid collisions depending on the locations of other vessels. Since this effect is taken into consideration in modeling casualties, we keep these movements independent to simplify the analysis. One would expect a strong and beneficial dependence of the navigation errors due to the common effect of currents. Therefore, the independence assumption yields a rather pessimistic prediction for the casualties.

At each time step, a vessel located at a specific grid travels into one of the grids above or below depending on its location and its direction. For example, if a vessel is travelling in the north-to-south direction and currently located at $(i, j)$ then it can be found in the southern grid $(i-1, j)$, in the southwestern $(i-1, j-1)$, or in the southeastern grid $(i-1, j+1)$ relative to its previous location in the next time period. Since, a vessel travels from one grid to another neighbor grid during $\Delta t$, the size of the grids and $\Delta t$ depend on the size and the average speed of the vessels. Figure 8 below shows a vessel located at $(i, j)$ in a rectangular grid, and possible transitions. 


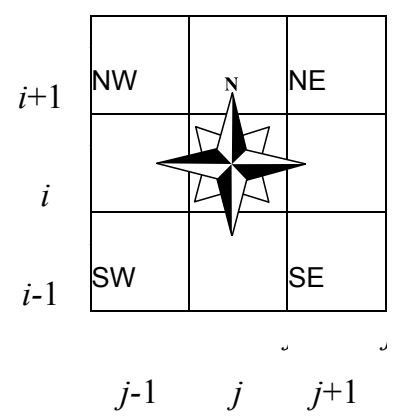

Figure 8. Possible transitions of a vessel located at $(i, j)$ in a rectangular grid

Since during each time step, a vessel travels one grid upward or downward depending on its route, the location of the vessel also determines the time that the vessel entered the waterway. That is if the current state of a vessel is $(i, j)$ at time $t$ and if this vessel is traveling upward, then it can be deduced that this vessel entered the waterway at $(1, \cdot)$ at time $t-i$. Similarly, if the current state of a vessel is $(i, j)$ at time $t$ and if this vessel is travelling downward, then it can be deducted that this vessel entered the waterway at $(n, \cdot)$ at time $t-(n-i)$. Therefore the superscript $n$ is omitted in $X_{k}^{n}(t)$ and the state of the system is simply denoted as $X_{k}(t)$ in the remaining part of the paper.

The state-transition probabilities at time $t$ can be written as

$$
\begin{aligned}
& P\left[X_{\mathrm{U}}(t+1)=(i+1, j-1) \mid X_{\mathrm{U}}(t)=(i, j)\right]=p_{\mathrm{NW}}(i, j) \\
& P\left[X_{\mathrm{U}}(t+1)=(i+1, j) \mid X_{\mathrm{U}}(t)=(i, j)\right]=p_{\mathrm{N}}(i, j) \\
& P\left[X_{\mathrm{U}}(t+1)=(i+1, j+1) \mid X_{\mathrm{U}}(t)=(i, j)\right]=p_{\mathrm{NE}}(i, j) \\
& P\left[X_{\mathrm{D}}(t+1)=(i-1, j-1) \mid X_{\mathrm{D}}(t)=(i, j)\right]=p_{\mathrm{SW}}(i, j) \\
& P\left[X_{\mathrm{D}}(t+1)=(i-1, j) \mid X_{\mathrm{D}}(t)=(i, j)\right]=p_{\mathrm{S}}(i, j) \\
& P\left[X_{\mathrm{D}}(t+1)=(i-1, j+1) \mid X_{\mathrm{D}}(t)=(i, j)\right]=p_{\mathrm{SE}}(i, j)
\end{aligned}
$$

where $(i, j)$ take values in appropriate limits according to the boundary conditions and the topology of the waterway. In the above equations, $p_{d}(i, j)$ is the probability that a vessel located at grid $(i, j)$, will continue its route in direction $d \in\{\mathrm{NW}, \mathrm{N}, \mathrm{NE}, \mathrm{SW}, \mathrm{S}, \mathrm{SE}\}$ in the next $\Delta t$ time units. The drift model explained in Section 5 provides these drift probabilities.

The probability that a vessel travelling in direction $k \in\{\mathrm{U}, \mathrm{D}\}$, is located at grid $(i, j)$ at time $t+1$ can be written conditioned on its location at $t$ as

$$
\begin{aligned}
P\left[X_{\mathrm{U}}(t+1)=(i, j)\right] & =p_{\mathrm{NE}}(i-1, j-1) P\left[X_{\mathrm{U}}(t)=(i-1, j-1)\right] \\
& +p_{\mathrm{N}}(i-1, j) P\left[X_{\mathrm{U}}(t)=(i-1, j)\right] \\
& +p_{\mathrm{NW}}(i-1, j+1) P\left[X_{\mathrm{U}}(t)=(i-1, j+1)\right] \\
P\left[X_{\mathrm{D}}(t+1)=(i, j)\right]= & p_{\mathrm{SE}}(i+1, j-1) P\left[X_{\mathrm{D}}(t)=(i+1, j-1)\right] \\
& +p_{\mathrm{S}}(i+1, j) P\left[X_{\mathrm{D}}(t)=(i+1, j)\right]
\end{aligned}
$$




$$
+p_{\mathrm{SW}}(i+1, j+1) P\left[X_{\mathrm{D}}(t)=(i+1, j+1)\right]
$$

Let $\pi_{i, j}^{k}(t)=P\left[X_{k}(t)=(i, j)\right]$ and $\underline{\pi}_{i}^{k}(t)=\left[\pi_{i, 1}^{k}(t), \pi_{i, 2}^{k}(t), \ldots, \pi_{i, M}^{k}(t)\right]$. Then equation (14) can be written in matrix form as

$$
\begin{aligned}
& \underline{\pi}_{i}^{\mathrm{U}}(t+1)=\underline{\pi}_{i-1}^{\mathrm{U}}(t) Q_{i}^{\mathrm{U}} \quad i=2,3, \ldots, N-1 \\
& \underline{\pi}_{i}^{\mathrm{D}}(t+1)=\underline{\pi}_{i+1}^{\mathrm{D}}(t) Q_{i}^{\mathrm{D}} \quad i=N-1, N-2, \ldots, 1
\end{aligned}
$$

where matrices $Q_{i}^{k}$ are the state transition matrices of the horizontal drift of a vessel located at vertical location $i$ travelling in direction $k$ with elements $p_{d}(i, j)$ or 0 .

Given $\underline{\pi}_{1}^{\mathrm{U}}(\tau)$ and $\underline{\pi}_{N}^{\mathrm{D}}(\tau)$, equations (15) and (16) can be used to determine $\underline{\pi}_{N}^{k}(\tau+t)$ for $t=1,2, \ldots$ and $k \in\{\mathrm{U}, \mathrm{D}\}$ recursively. The vectors $\underline{\pi}_{1}^{\mathrm{U}}(\tau)$ and $\underline{\pi}_{N}^{\mathrm{D}}(\tau)$ are completely determined by the arrival process of the vessels.

\subsection{Modeling of the Vessel Arrival Process}

Considering all the vessels departing from different locations and arriving the waterway at a given time, we assume that the number of tankers arriving in a time period $[0, t)$ is a Poisson random variable with mean $\lambda_{k} t$ where $\lambda_{\kappa}$ is the arrival rate of tankers (number of tankers arriving per unit time) travelling in direction $k, k \in\{\mathrm{U}$, D \}. Therefore for a small $\Delta t$, the probability that there will be a new arrival is $r_{k}=\lambda_{k} \Delta t$ and the probability that there will be no arrivals is $1-r_{k}=1-\lambda_{k} \Delta t$. Namely, at a given time step, a vessel enters a waterway going up with probability $r_{\mathrm{U}}$ or enters going down with probability $r_{\mathrm{D}}$.

Let $\underline{h}_{\mathrm{k}}$ be a $1 \times M$ vector whose $j$ th element is the probability that a vessel travelling in direction $k$ enters the waterway at horizontal location $j$. Then, for a vessel arriving the waterway at time $t$

$$
\begin{aligned}
& \underline{\pi}_{1}^{\mathrm{U}}(t)=r_{\mathrm{u}} \underline{h}_{\mathrm{u}} \quad \forall t \\
& \underline{\pi}_{N}^{\mathrm{D}}(t)=r_{\mathrm{D}} \underline{h}_{\mathrm{D}} \quad \forall t
\end{aligned}
$$

Figures 9 and 10 depict the complete state transition diagrams for a vessel travelling up and down respectively. In these state transition diagrams, S0 and N0 are the states of a vessel located outside a waterway and $\mathrm{S} 0$ is the southward and N0 is the northward state. 


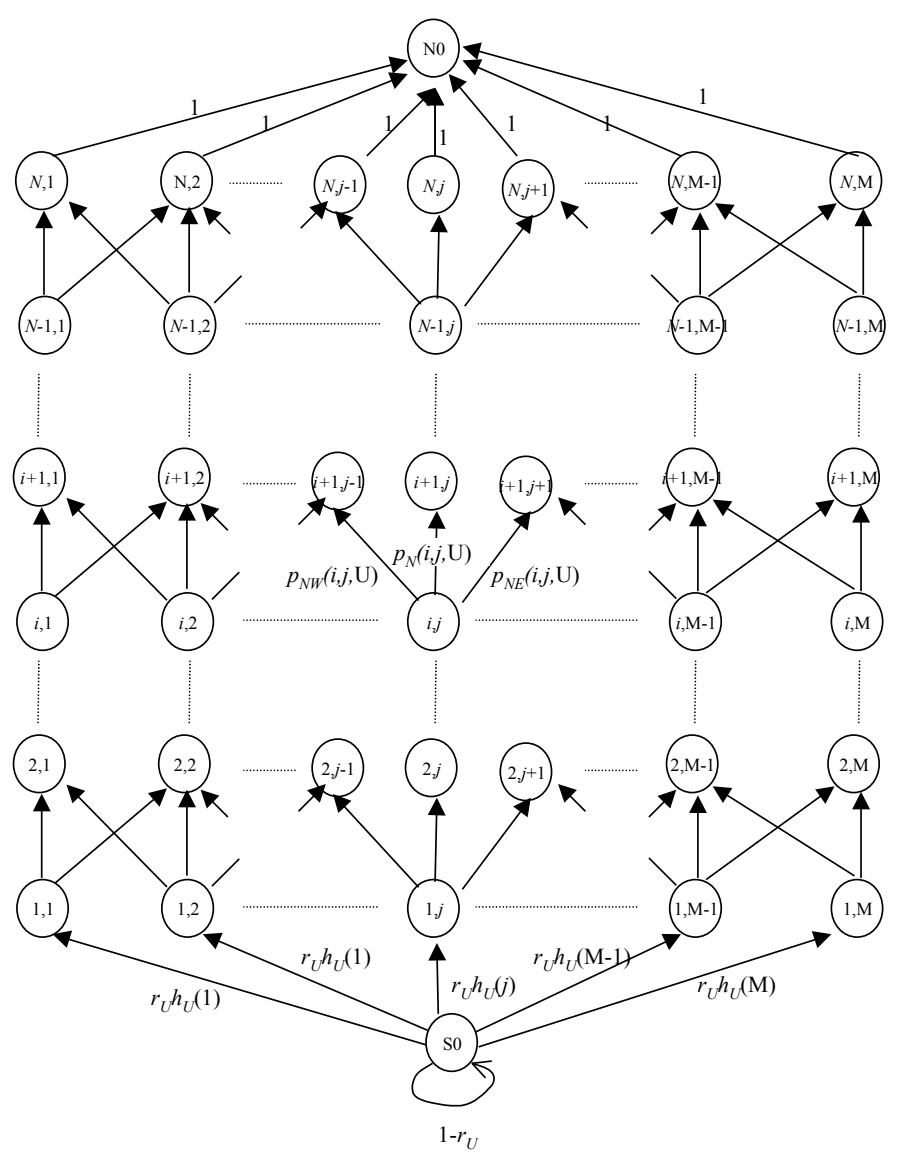

Figure 9. State transition diagram for the movements of a vessel traveling up along a waterway

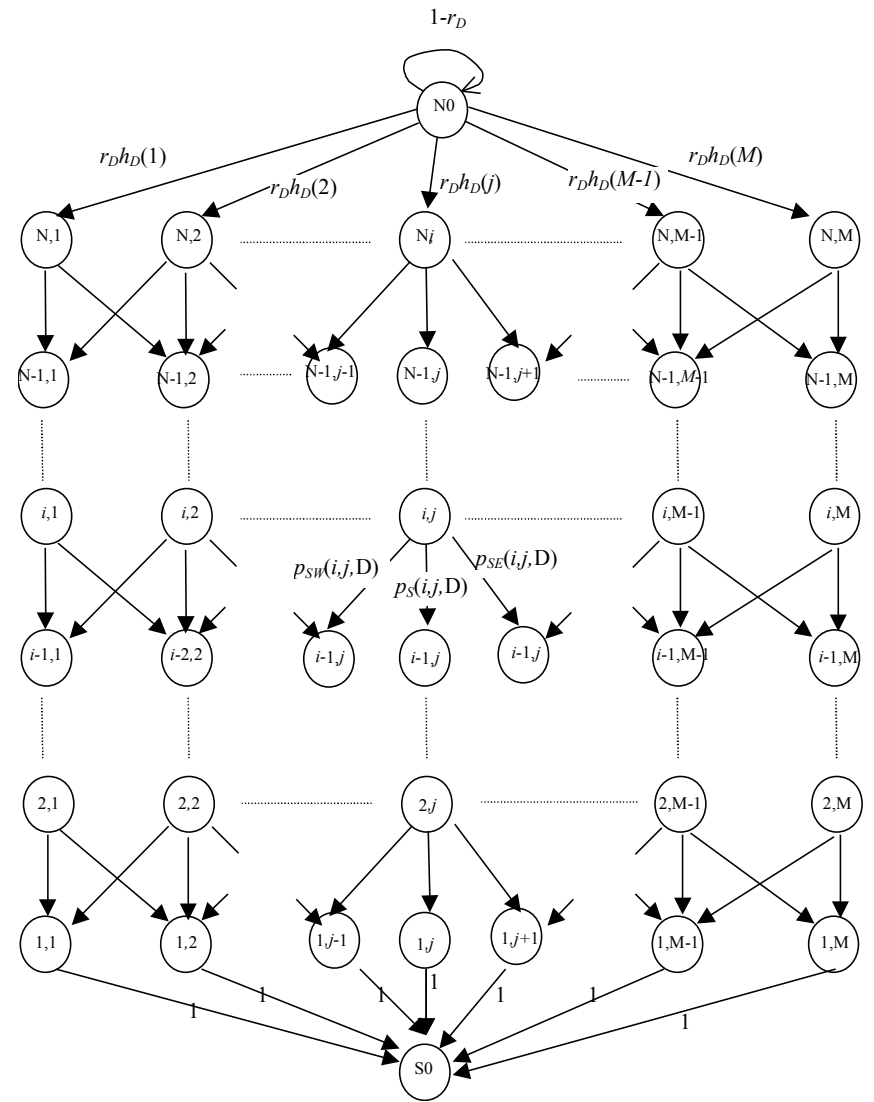

Figure 10. State transition diagram for the movements of a vessel traveling down along a waterway 


\subsection{Modeling of Casualties}

A casualty is defined as a collision of two vessels or grounding of a vessel. In this study, we focus on both intervessel collision and also grounding. Figure 11 shows the rectangular grid, arrival model, and casualties.

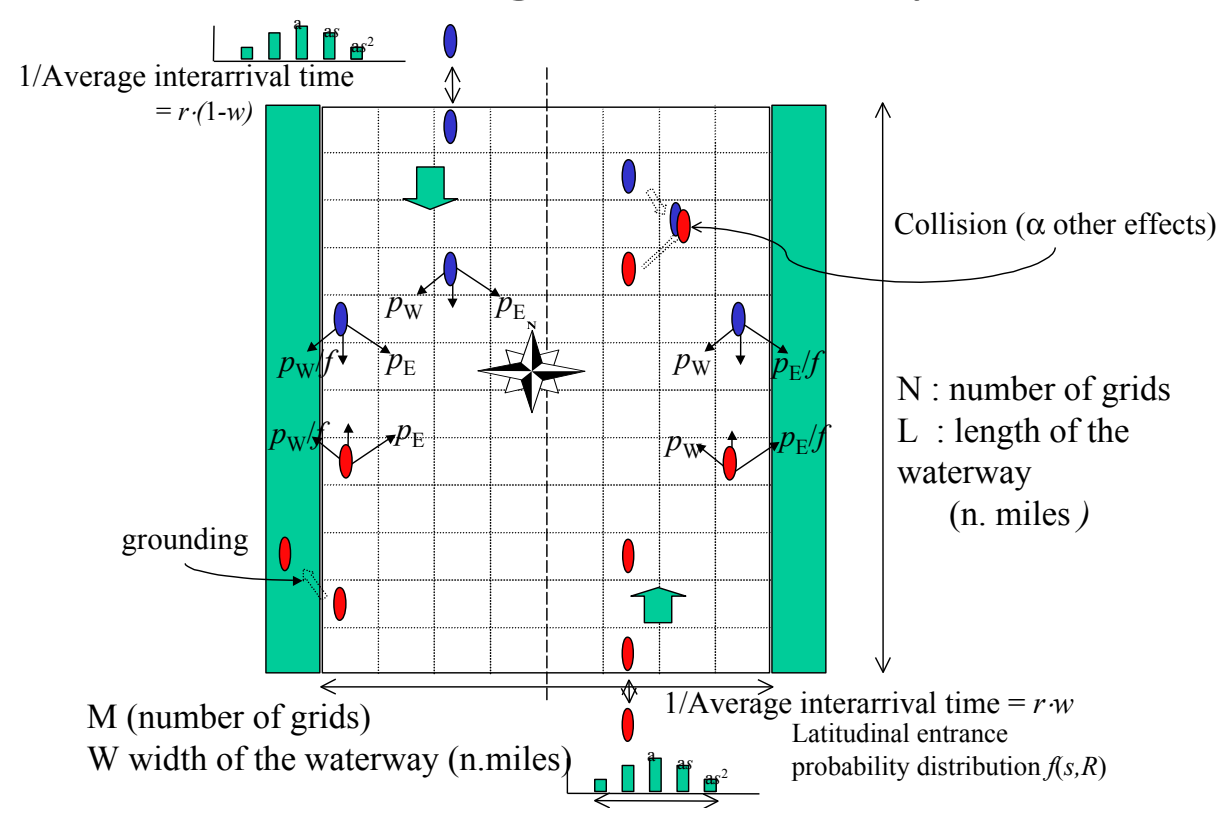

Figure 11. Modeling of vessel traffic and casualties

\section{Modeling of Intervessel Collisions:}

According to the state-space model developed above, an intervessel collision may occur when two vessels arrive in the same grid at the same time step. As mentioned before, all tankers travelling through a waterway are required to maintain a constant speed, say $v_{s}$ that is $10 \mathrm{nmiles} / \mathrm{hr}$ for the Bosphorus. Furthermore, it is not allowed for a vessel to pass another one which travels ahead. Therefore an intervessel collision is a result of the arrivals of two vessels travelling up and down in a given grid. Let $C_{i, j}(t)$ be an indicator random variable which is 1 if there is a collision at location $(i, j)$ due to entry of two vessels travelling up and down respectively at time $t$ and 0 otherwise.

Maritime regulations dictate boats to turn right immediately when they see a boat travelling towards them. If they can complete the necessary manoeuvres in time, there will be no collision. Because of human errors, visibility, machine failures, etc., a ship may not complete its manoeuvre in time and there will be a collision. Let $\alpha$ be the conditional probability that two vessels collide given that these vessels are located at the same grid at the same time, i.e.,

$$
\alpha=P\left[C_{i, j}(t)=1 \mid X_{\mathrm{U}}(t)=(i, j) \text { and } X_{\mathrm{D}}(t)=(i, j)\right] \quad \forall t, \quad i, j \in A_{C}
$$

where $A_{C}$ is the set of grids that an intervessel collision can take place. Once can set $\alpha=1$ to obtain conservative, or pessimistic, estimates of the expected number of casualties. Alternatively, past data on the causes of intervessel collisions or expert opinion can be used to estimate this conditional probability.

Then the probability of collision at location $(i, j)$ at time $t$ can be written as 


$$
P\left[C_{i, j}(t)=1\right]=P\left[C_{i, j}(t)=1 \mid X_{\mathrm{U}}(t)=(i, j) \text { and } X_{\mathrm{D}}(t)=(i, j)\right] P\left[X_{\mathrm{U}}(t)=(i, j) \text { and } X_{\mathrm{D}}(t)=(i, j)\right]
$$

Since the vessel movements are assumed to be independent of each other, the above equation yields

$$
P\left[C_{i, j}(t)=1\right]=\alpha P\left[X_{\mathrm{U}}(t)=(i, j)\right] P\left[X_{\mathrm{D}}(t)=(i, j)\right]
$$

Equivalently in matrix notation

$$
P\left[C_{i, j}(t)=1\right]=\alpha \underline{\pi}_{i}^{\mathrm{U}} e_{j} \quad \underline{\pi}_{i}^{\mathrm{D}} e_{j}
$$

where $e_{j}$ is a column vector whose $j$ th element is 1 and all the other elements are zero. Note that the above equation captures the effects of vessel dimensions embedded in the terms of $\underline{\pi}_{1}^{\mathrm{U}}$ and $\underline{\pi}_{N}^{\mathrm{D}}$.

Let $N_{\mathrm{C}}(t)$ be the number of collisions along the waterway at time $t$. Considering all the vessels that are located in other grids, the expected number of collisions at time $t, E\left[N_{\mathrm{C}}(t)\right]$ can be written as

$$
E\left[N_{C}(t)\right]=\sum_{(i, j) \in A_{C}} P\left[C_{i, j}(t)=1\right]
$$

\section{Modeling of Grounding:}

Another type of casualty is grounding. In our model when a vessel moves into a grid located in the grounding zone, the vessel may be grounded. When a vessel moves into a grounding zone, it first tries to move away from grounding by some manoeuvres. Similar to the previous case, because of human factors, machine failures, visibility, etc., these manoeuvres may not protect the vessel from grounding.

Let $G_{i, j}(t)$ be an indicator random variable which is 1 if there is a grounding at location $(i, j)$ at time $t$ and 0 otherwise. Let $\beta$ be the conditional probability that a vessel is grounded given that this vessel moves into a grid located in the grounding zone, i.e.,

$$
\beta=P\left[G_{i, j}(t)=1 \mid X_{\mathrm{k}}(t)=(i, j)\right] \quad \forall t, k \quad(i, j) \in A_{G}
$$

where $A_{G}$ is the set of grids that a grounding can take place.

Since a grounding can be caused by a vessel travelling in either direction, the probability of collision at location $(i, j)$ at time $t$ can be written as

$$
\begin{aligned}
P\left[G_{i, j}(t)=1\right] & =P\left[G_{i, j}(t)=1 \mid X_{\mathrm{U}}(t)=(i, j)\right] P\left[X_{\mathrm{U}}(t)=(i, j)\right]+P\left[G_{i, j}(t)=1 \mid X_{\mathrm{D}}(t)=(i, j)\right] P\left[X_{\mathrm{D}}(t)=(i, j)\right] \\
& =\beta\left(P\left[X_{\mathrm{U}}(t)=(i, j)\right]+P\left[X_{\mathrm{D}}(t)=(i, j)\right]\right) \quad(i, j) \in A_{G}
\end{aligned}
$$

or in matrix notation

$$
P\left[G_{i, j}(t)=1\right]=\beta \underline{\pi}_{i}^{\mathrm{U}} e_{j}+\underline{\pi}_{i}^{\mathrm{D}} e_{j}
$$

Let $N_{\mathrm{G}}(t)$ be the number of groundings along the waterway at time $t$. Considering all the vessels that are located in other grids, the expected number of groundings at time $t, E\left[N_{\mathrm{G}}(t)\right]$ can be written as

$$
E\left[N_{G}(t)\right]=\sum_{(i, j) \in A_{G}} P\left[G_{i, j}(t)=1\right]
$$


Finally the expected number of casualties along the waterway at time $t E[N(t)]$ is the sum of the expected number of collisions and groundings, i.e.,

$$
E[N(t)]=E\left[N_{C}(t)\right]+E\left[N_{G}(t)\right]
$$

In the next section, we determine the steady-state casualty probabilities and expected number of casualties.

\section{Steady-State Analysis of Vessel Casualties}

In the hydrodynamic model and drift probability model, we used long-term variability distributions of the parameters. More specifically, we represented wind variability and vessel type variability by their long-term variability distributions. Therefore, analysis of steady-state vessel casualties is of interest.

In order to obtain steady-state results, we start our analysis from vessel traffic equations. By using Equation (17), equation (15) can be written in terms of $\underline{\pi}_{1}^{\mathrm{U}}(t)$ as

$$
\underline{\pi}_{i}^{\mathrm{U}}(t+1)=\underline{\pi}_{1}^{\mathrm{U}}(t) \prod_{k=2}^{i} Q_{k}^{\mathrm{U}}=w r \underline{h}_{\mathrm{U}} \prod_{k=2}^{i} Q_{k}^{\mathrm{U}} \quad i=2,3, \ldots, N-1
$$

where $r$ is the total arrival rate of the vessels, $r=r_{u}+r_{D}$ and $w$ is the fraction of the vessels entering the waterway in upward direction, $w=r_{u} /\left(r_{u}+r_{D}\right)$.

Note that the above expression for $\underline{\pi}_{i}^{\mathrm{U}}(t+1)$ is independent of $t$. Therefore, the steady state probability vector that a vessel travelling up will be located at $(i, j), j=1,2, \ldots, M$ is

$$
\lim _{t \rightarrow \infty} \underline{\pi}_{i}^{\mathrm{U}}(t)=w r \underline{h}_{\mathrm{U}} \prod_{k=2}^{i} Q_{k}^{\mathrm{U}}
$$

Similarly, Equation (18) and equation (16) yield

$$
\underline{\pi}_{i}^{\mathrm{D}}(t+1)=\underline{\pi}_{N}^{\mathrm{D}}(t) \prod_{k=i}^{N-1} Q_{k}^{\mathrm{D}}=(1-w) r \prod_{k=i}^{N-1} Q_{k}^{\mathrm{D}}
$$

The above expression for $\underline{\pi}_{i}^{\mathrm{D}}(t+1)$ is also independent of $t$. Therefore, the steady state probability vector that a vessel travelling down will be located at $(i, j), j=1,2, \ldots, M$ can be written as

$$
\lim _{t \rightarrow \infty} \pi_{i}^{\mathrm{D}}(t)=(1-w) r \prod_{k=i}^{N-1} Q_{k}^{\mathrm{D}}
$$

Since the steady-state probabilities for the location of vessels travelling up or down are available, equation (22) yields the steady state casualty probability at $(i, j)$ at time $t$ given as

$$
\lim _{t \rightarrow \infty} P\left[C_{i, j}(t)=1\right]=\alpha w(1-w) r^{2}\left[\underline{h}_{\mathbf{U}} \prod_{k=2}^{i} Q_{k}^{\mathbf{U}} e_{j}\right]\left[\underline{h}_{\mathbf{D}} \prod_{k=i}^{N-1} Q_{k}^{\mathbf{D}} e_{j}\right]
$$

and equation (23) gives the expected number of collisions at a given time in the long run given as 


$$
\lim _{t \rightarrow \infty} P\left[N_{c}(t)\right]=r^{2} w(1-w) \alpha \sum_{(i, j) \in A_{c}}\left[\underline{h}_{\mathbf{U}} \prod_{k=2}^{i} Q_{k}^{\mathbf{U}} e_{j}\right]\left[\underline{h}_{\mathbf{D}} \prod_{k=i}^{N-1} Q_{k}^{\mathbf{D}} e_{j}\right]
$$

Similarly, the steady-state probability of grounding at $(i, j)$ at time $t$ and the expected number of groundings in the long run follow equations (26) and (27) respectively:

$$
\begin{aligned}
& \lim _{t \rightarrow \infty} P\left[G_{i, j}(t)=1\right]=r \beta\left[w \underline{h}_{\mathbf{U}} \prod_{k=2}^{i} Q_{k}^{\mathbf{U}} e_{j}+(1-w) \underline{h}_{\mathbf{D}} \prod_{k=i}^{N-1} Q_{k}^{\mathbf{D}} e_{j}\right] \\
& \lim _{t \rightarrow \infty} E\left[N_{G}(t)\right]=r \beta \sum_{(i, j) \in A_{G}}\left[w \underline{h}_{\mathbf{U}} \prod_{k=2}^{i} Q_{k}^{\mathbf{U}} e_{j}+(1-w) \underline{h}_{\mathbf{D}} \prod_{k=i}^{N-1} Q_{k}^{\mathbf{D}} e_{j}\right]
\end{aligned}
$$

Summing up the expected number of intervessel collisions and groundings in the long run gives the expected total number of casualties at a given time in the long run which is

$$
\begin{gathered}
\lim _{t \rightarrow \infty} E[N(t)]=r^{2} w(1-w) \alpha \sum_{(i, j) \in A_{c}}\left[\underline{h}_{\mathbf{U}} \prod_{k=2}^{i} Q_{k}^{\mathbf{U}} e_{j}\right]\left[\underline{h}_{\mathbf{D}} \prod_{k=i}^{N-1} Q_{k}^{\mathbf{D}} e_{j}\right] \\
+r \beta \sum_{(i, j) \in A_{G}}\left[w \underline{h}_{\mathbf{U}} \prod_{k=2}^{i} Q_{k}^{\mathbf{U}} e_{j}+(1-w) \underline{h}_{\mathbf{D}} \prod_{k=i}^{N-1} Q_{k}^{\mathbf{D}} e_{j}\right]
\end{gathered}
$$

Note that the expected number of collisions in the long run can be written as $r^{2}$ times a constant and the expected number groundings can be written as $r$ times another constant. These constants reflect the effects of channel geometry, physical forcing mechanisms, etc. In other words as the vessel arrival rate increases, expected number of collisions and therefore the expected number of casualties increase by the square of the increase in arrival rate.

A further notice is due to the calculation of $r$. Remember that $r=\lambda \Delta t$ and $\Delta t$ is determined from the cell length and vessel speed. One may conjecture that as $\Delta t$ approaches zero, the expected number of collisions and groundings approach zero. However, since the number of arrivals is Poisson random variable, taking the limit as $\Delta t$ approaches 0 yield a continuous time Markov chain model with non-zero limiting probabilities and transient probabilities functions of $\mathrm{e}^{-\lambda t}$.

\section{Analysis of a Special Case}

In this section, we analyze a special case in order to exploit the analytical results presented in the previous section. We consider a $100 \times 10$ rectangular grid. It is assumed that intervessel collision can take place at $(\mathrm{i}, \mathrm{j}) \in A_{\mathrm{c}}, A_{\mathrm{c}}=\{(i, j) \mid i=2,3, \ldots, 99 ; j=2,3, \ldots, 8\}$ and a vessel may ground only at boundaries, i.e., at $j=1$ or $j=10$. A vessel may drift towards east with probability 0.1 and towards west with probability 0.1 when it is located in interior grids. When it reaches the grounding zone it stays there with probability 0.9 and returns to the interior region with probability 0.1 . Vessels arrive in the waterway with equal rates from North or from South and enter the waterway from the center zone randomly.

This special case can be solved semi-symbolically. The solution, i.e., the expressions for the casualty probabilities and steady-state expected number of collisions and groundings are not given here for brevity. We 
investigate the effects of arrival rate of vessels, those of waterway length and width, and also the effects of drift probability.

Figure 12 shows the effects of vessel arrival rate. As mentioned above, the expected number of collisions and casualties are second degree functions of the arrival rate while the expected number of groundings is a linear function of the arrival rate. Figure 13 depicts the effects of the length of the waterway. These figures support the intuition that as the waterway length increases, it is more likely that an intervessel collision or a grounding takes place. The effects of waterway width are shown in Figure 14. As the waterway width increases, it is less likely that two vessels will be located at the same location at the same time, thus the expected number of collisions decreases. Similarly, as the waterway width increases, it is less likely that a vessel will drift from its intended route that much to reach the grounding zone and therefore the expected number of groundings also decreases. As a result, the expected number of casualties decreases as the waterway width increases. Finally, Figure 15 depicts the effects of the drift probability. As expected, as it becomes more likely that a vessel drifts away from its intended route, it becomes more likely that it collides into another vessel or grounds. Therefore, the expected number of casualties increases as the drift probabilities increase.
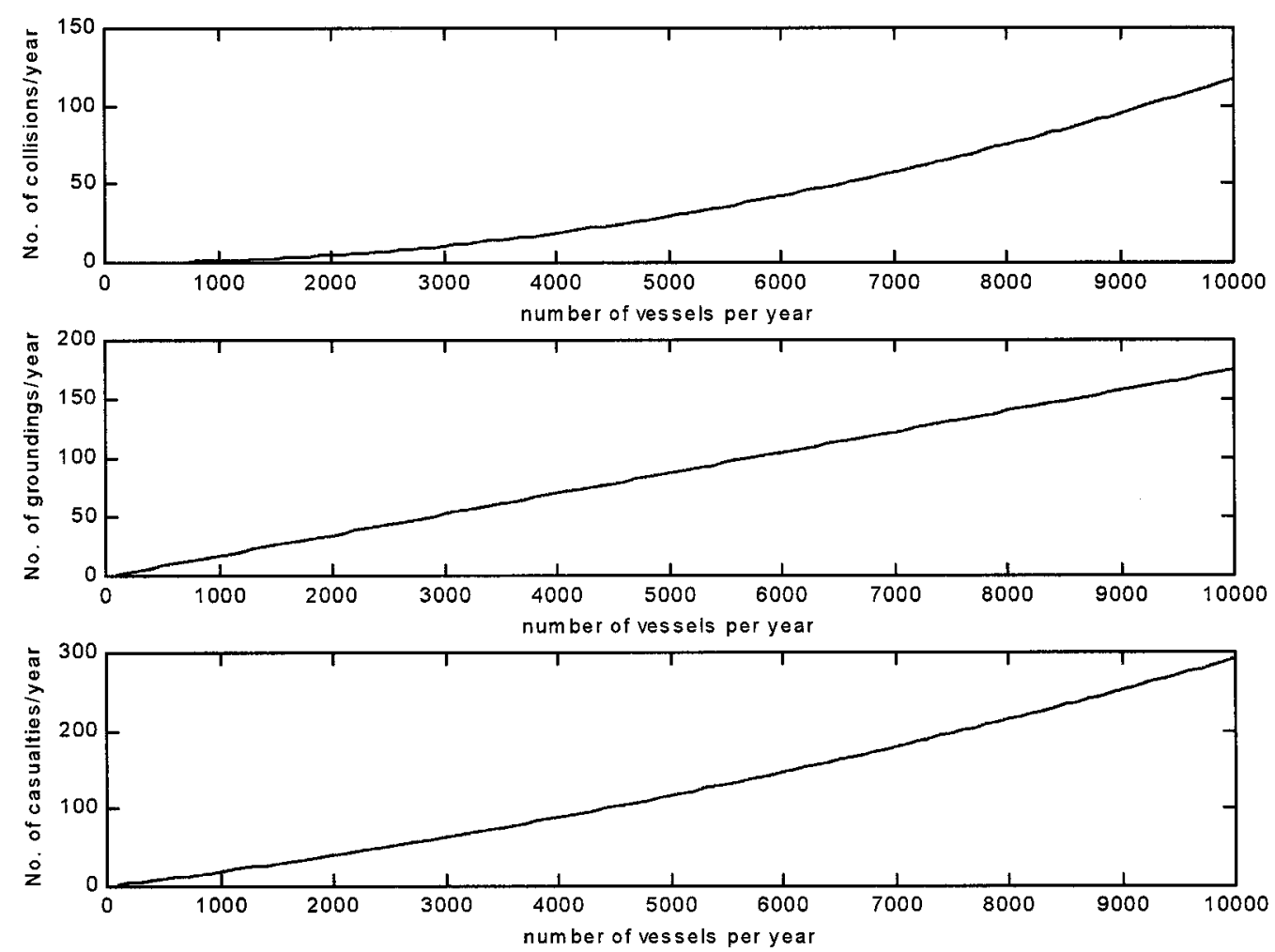

Figure 12. Effect of traffic intensity 

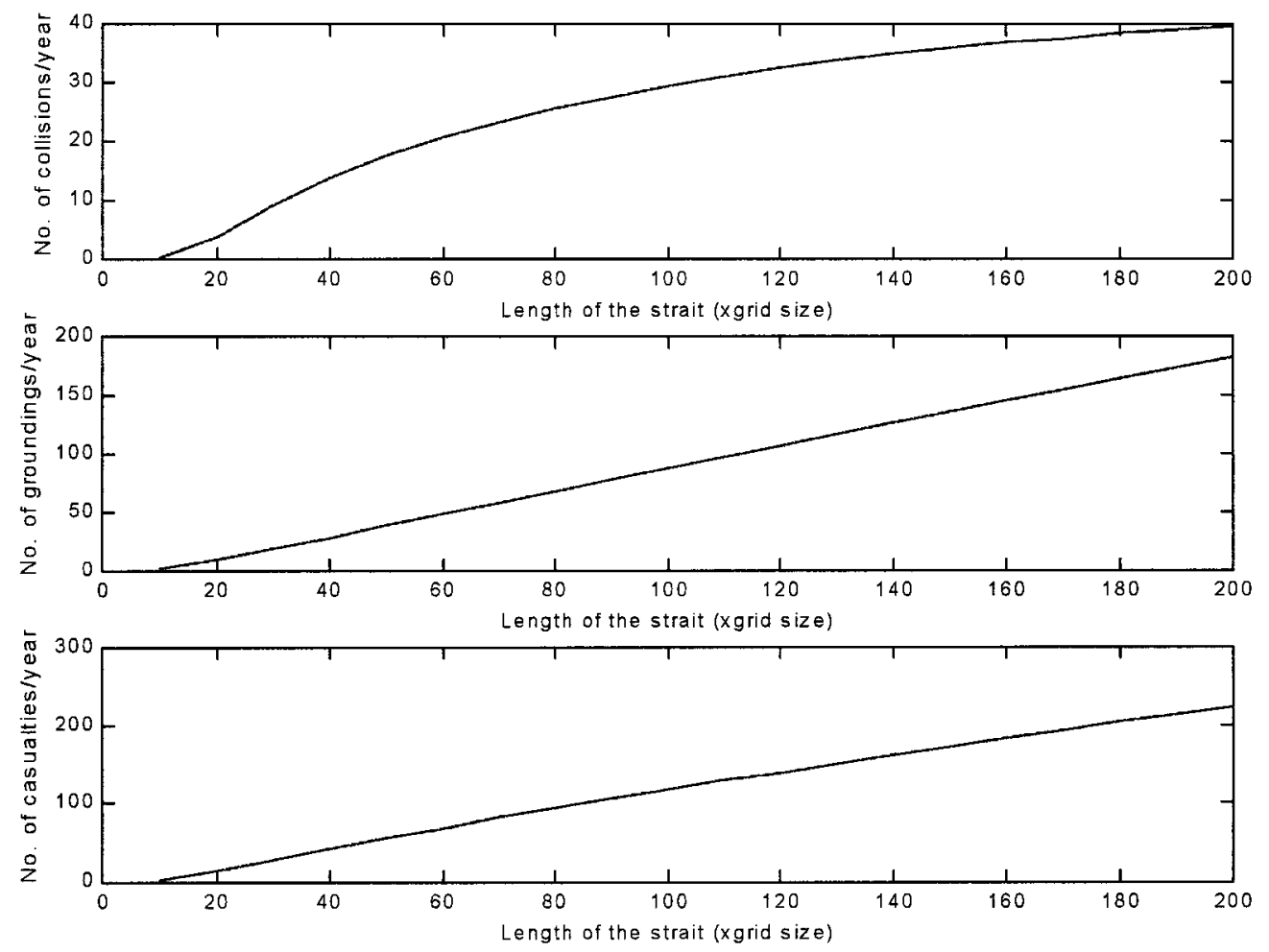

Figure 13. Effect of waterway length
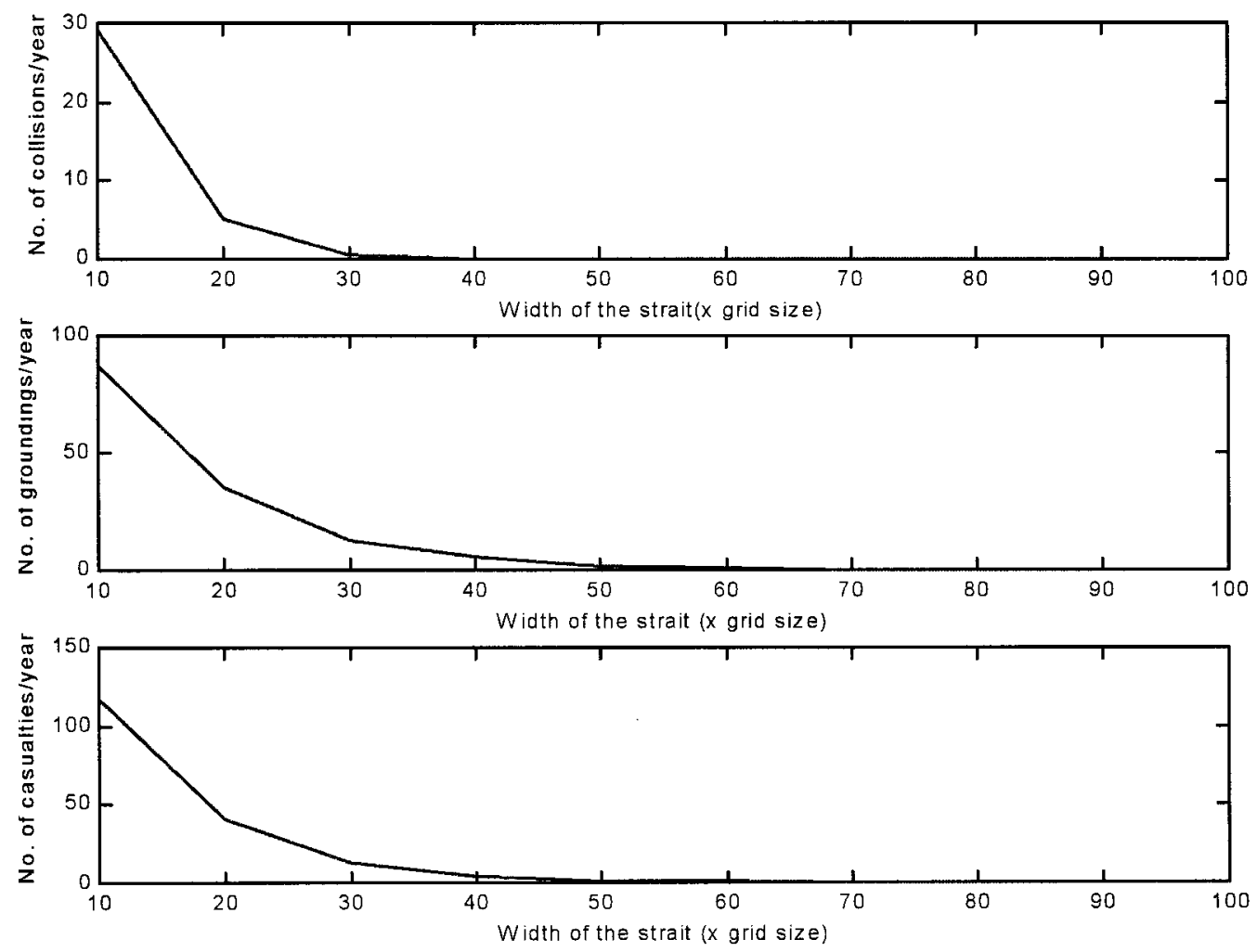

Figure 14. Effects of waterway width 

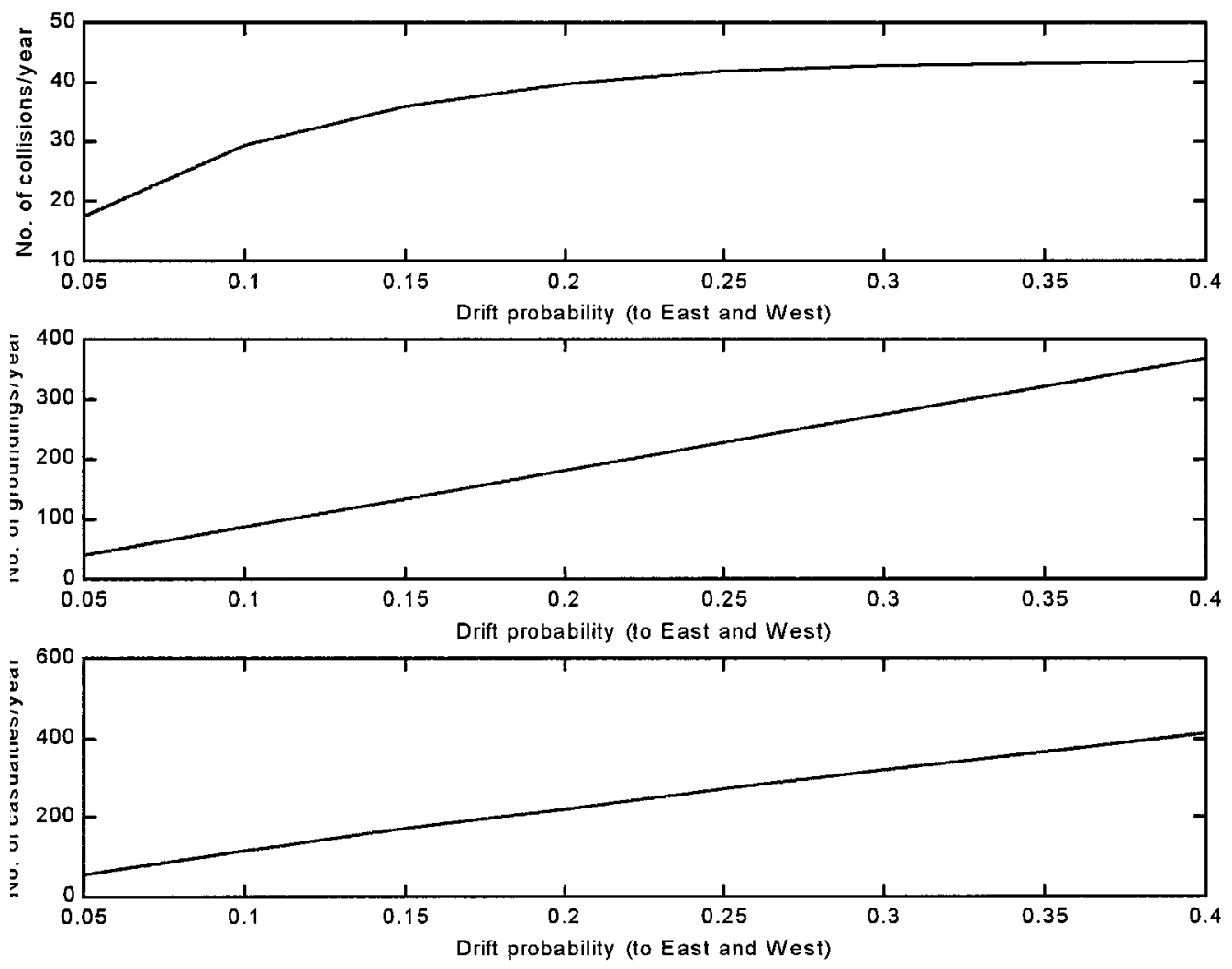

Figure 15. Effects of drift probability

\section{Conclusions}

In this study, we present a physics-based stochastic model to evaluate the vessel casualty risks resulting from tanker traffic through narrow waterways. We believe that there are three contributions of this work. As a contrast to the previous studies based on statistical analysis of past data, the model we present is based on physical modeling of forcing mechanisms, i.e., modeling of currents and waves, and modeling of movements of individual ships. Therefore, the approach we present is geared towards explaining why vessels collide at a specific waterway at a specific time. In this physics-based model, we determined the transition probabilities of the underlying Markov chain by using two physical models, one that models the surface currents, and another one that models the drift of a tanker under the effects of these currents. These models are solved numerous times for each different realization of input parameters to determine the drift angle distribution by Monte Carlo simulation. We also believe that linking these models to a Markov chain model by utilizing Monte Carlo simulation is a novel approach.

As a second contribution of this work, we derived an analytic formula that relates the accident risk to the vessel traffic. More specifically, we showed that as the vessel arrival rate increases, the expected number of collisions and therefore the expected number of casualties increase by the square of the arrival rate. This result also emphasizes the difference between the previous studies based on statistical analysis of past data and our method. The vessel arrival rate is one of the independent parameters used to estimate the number of casualties in multiple regression models previously developed for narrow channels, e.g, (Maio, et. al., 1991). Thus the estimated coefficient of the arrival rate impose a linear relationship between arrival rate and the number of casualties. Although this linear approximation can be accurate in a given range of the arrival rate, it looses its 
accuracy, if the arrival rate is increased. This is the situation, for example, for the Bosphorus. The oil tanker traffic through the Bosphorus Strait is expected to increase as a result of exploitation of oil reserves in the former-Russian republics and there are no historical data to build a regression model to estimate the effects of this increase on the accident risk..

Finally, an experimental model is developed and applied to a hypothetical waterway. This experimental model and its analysis shows the possible outputs that can be obtained when this methodology is applied to a given waterway.

The results of this model can be used both in traffic control by tracking individual ships and then using the transient analysis to estimate possible casualties and also in planning and policy development by focusing on steady-state behavior. Furthermore, this methodology can also be used for the real-time simulation of vessel traffic. That is, if the positions of the vessels on a waterway can be determined at a given time, say by using a geographical positioning system, we can use the methodology presented in this study to determine the expected positions of the vessels in a given time interval and control the traffic to minimize the risk of potential casualty.

As stated before, this study should be seen as a first step towards the modeling and analysis of vessel casualties resulting from tanker traffic through a narrow waterway, for example, through the Bosphorus. Developing a complete and also accurate model for a waterway requires considerable time and effort to collect reliable data, to fine-tune the physical models according to the specifics of the waterway under consideration, and to analyze the results.

\section{Acknowledgment}

We thank the anonymous referees for several suggestions that helped to improve the quality of this paper.

\section{References}

Anderson, E.; Talley, W. (1995), "The oil spill size of tanker and barge accidents: Determinants and policy implications," Land Economics, Vol: 71, Iss: 2, p: 216-228.

Cohen, M. J. (1995), “Technological disasters and natural resource damage assessment: An evaluation of the Exxon Valdez oil spill,” Land Economics, Vol: 71, Iss: 1, p: 65-82.

Daily, J.W., Harleman, D.R.F. (1966), Fluid Dynamics, Addison Wesley, New York.

Etkin, D.S. (1997), Oil spills from vessels (1960-1995): An international historical perspective, Oil Spill Intelligence Report, Cutter Information Corp. Arlington, USA.

Kornhauser, A.L, Clark, W.A. (1995), "Quantitative forecast of vessel casualties resulting from additional oil tanker traffic through the Bosphorus,” ALK Associates Inc. Report, September, Princeton, NJ.

Lamb, H. (1945), Hydrodynamics: $6^{\text {th }}$ edition, Dover, New York.

Le Blanc, L. A; Rucks, C.T. (1995), “A cluster analysis of vessel accidents,” Logistics \& Transportation Review, Vol: 31, Iss: 1, p: 47-62.

Maio, D., Ricci, R., Rossetti, M., Schwenk, J., Liu, T. (1991), Port Needs Study, Vol. 1, Rep. No. DOT-CG-N01-91-1.2, USDOT/RSPA/VolpeTSC 
Oğuzülgen, Saim (1995), "The importance of pilotage services in the Turkish straits for the protection of life, property, and the environment," in: Turkish Straits: New Problems and New Solutions, pp.108-126, ISIS Ltd., Istanbul.

Oshins, A. H. (1992), “The Risk of Oil Spills,” Risk Management, Vol: 39, Iss: 12, p: 54.

Shapiro, S.; Huntley, G. (1989) “Exxon's Spill cover totals \$600 Million; Oil Spill Illustrates Risk Management Flaws.” Business Insurance, Vol: 23 Iss: 14 Date: Apr 3, 1989 p: 1, 45-46.

Talley, W. (1995a), "Vessel damage severity of tanker accidents," Logistics \& Transportation Review, Vol: 31, Iss: 3, p: 191-207

Talley, W. K (1995b), "Safety investments and operating conditions: Determinants of accident passenger-vessel damage cost,” Southern Economic Journal, Vol: 61, Iss: 3, p: 819-829.

Talley, W. K. (1996), "Determinants of cargo damage risk and severity: The case of containership accidents," Logistics \& Transportation Review, Vol: 32, Iss: 4, p: 377-388.

Tan, B. and Otay, E. (1998), “A stochastic model for vessel casualties resulting from oil tanker traffic through narrow waterways," Proceedings of the 1998 European Simulation Multiconference, Ed. R. Zobel and D.Moeller, pp. 881-885.

Unsworth, Edwin (1996), "Spill worsens as rescue delayed,” Business Insurance, Vol: 30 Iss: 9 Date: Feb 26, 1996 p: 17.

Unsworth, Edwin (1997), “Tanker spill costs still being tallied,” Business Insurance, Vol: 31 Iss: 2 Date: Jan 13, 1997 p: 45. 